\title{
Left atrial longitudinal strain in the contractile phase as a predictor of sinus rhythm maintenance after electrical cardioversion performed due to persistent atrial fibrillation
}

\author{
Paweł Wałek ${ }^{1,2}$, Urszula Grabowska33, Elżbieta Cieśla¹, Iwona Gorczyca,2, Beata Wożakowska-Kapłon ${ }^{1,2}$ \\ 1 Collegium Medicum, Jan Kochanowski University, Kielce, Poland \\ 2 1st Department of Cardiology and Electrotherapy, Voivodship Hospital Kielce, Poland \\ 3 Medical Laboratory, Voivodship Hospital Kielce, Poland
}

Correspondence to: Paweł Wałek, MD, PhD, 1st Department of Cardiology and Electrotherapy, Voivodship Hospital Kielce, ul. Grunwaldzka 45, 25-736 Kielce, Poland, phone: +48413671510 , email: pawel.walek@02.pl Received: January 23, 2021 Revision accepted: March 12, 2021.

Published online: March 25, 2021. Kardiol Pol. 2021; 79 (4): 458-460 doi:10.33963/KP.15913 Copyright by the Author(s), 2021
Introduction Atrial fibrillation (AF) is the most common persistent arrhythmia and one of the most significant cardiovascular risk factors. ${ }^{1}$ Direct current cardioversion (DCCV) is a procedure of choice to restore sinus rhythm (SR) in patients with persistent AF because it is readily available and cost effective.

Numerous studies are investigating risk factors of recurrent AF to better understand how DCCV contributes to SR maintenance. Among many new echocardiographic parameters assessing the prognosis of SR maintenance after DCCV, the prognostic value of left atrial (LA) strain (LAS) and peak LAS rate (pLASR) has been indicated. So far, most studies evaluated the suitability of using the LA wall strain in the prognosis of SR maintenance after DCCV, with a focus on the global strain in the reservoir phase. ${ }^{2-6}$ In this study, we used speckle-tracking echocardiography (STE) to measure LAS and pLASR in the reservoir, conduit, and contractile phases the day after effective DCCV. We assessed the prognostic value of LAS and pLARS in all phases regarding SR maintenance for 12 months after a successful DCCV. Previously, we analyzed this group of patients for prognostic value of left atrial wall dyskinesia in terms of maintaining SR after DCCV. ${ }^{7}$

Methods The study protocol was approved by the Institutional Review Board of the Świętokrzyskie Medical Chamber and informed consent was obtained from each patient.
We included 89 patients who underwent successful elective DCCV between November 2015 and August 2018 with ejection fraction during SR of $40 \%$ or greater and no moderate or severe valve disease. Left atrial wall deformation analysis was performed using STE during SR on the day after successful DCCV. We used the upslope of the $\mathrm{R}$ wave as the electrocardiography reference point for strain and pLASR measurements as recommended in the consensus document. ${ }^{8}$ Left atrial myocardial deformation assessed during SR after successful cardioversion was presented as the LA strain or as the peak strain rate during the reservoir phase (LASr or pLASRr), conduit phase (LAScd or pLASRcd), and contractile phase (LASct or pLASRct) in the apical 4-chamber (4c) and 2-chamber (2c) views, and the average of both views (mean) as recommended in the consensus document. ${ }^{8}$

Statistical analysis The results are presented as means (SD) and numbers (percentages). The predictors of SR maintenance were analyzed with univariate logistic regression. The stepwise multivariable logistic regression analysis included echocardiographic parameters assessing mechanical remodeling and left ventricular filling pressure with the lowest $P$ value evaluated in a univariate logistic analysis. The differences between the area under the curve (AUC) in the same echocardiographic views were compared using the $Z$ test. Statistical significance 
TABLE 1 Area under the curve comparisons for 12-month SR maintenance for strain and strain rate measurements in the 4-chamber and 2-chamber apical projections and mean results from both projections

\begin{tabular}{lll} 
Comparisons & AUC & $P$ value \\
\hline LASr4c vs LASct4c & 0.68 vs 0.765 & 0.1 \\
\hline pLASRr4c vs pLASRct4c & 0.563 vs 0.726 & 0.003 \\
\hline LASr2c vs LASct2c & 0.673 vs 0.669 & 0.92 \\
\hline pLASRr2c vs pLASRct2c & 0.605 vs 0.664 & 0.3 \\
\hline LASr mean vs LASct mean & 0.709 vs 0.734 & 0.58 \\
\hline pLASRr mean vs pLASRct mean & 0.59 vs 0.704 & 0.02 \\
\hline
\end{tabular}

Abbreviations: AUC, area under the curve; ct, contractile phase; 4c, 4-chamber; LAS left atrial strain; pLASR, peak left atrial strain rate; $r$, reservoir phase; $2 c, 2$-chamber;

was set at $P$ value of less than 0.05 . The statistical analyses were performed with the STATISTICA 13.3 software (TIBCO Software Inc., Tulsa, Oklahoma, United States). Detailed methodology, characteristics of the study group, and limitations of the study were described previously. ${ }^{7}$

Results and discussion After 12 months, 42 patients (47.2\%) maintained SR (Supplementary material, Tables S1 and S2). The univariate logistic regression analysis revealed several significant predictors of SR maintenance after 12 months of observation, including male sex (odds ratio [OR], 2.96; 95\% CI, 1.16-7.54; $P=0.02$ ), LA end-diastolic volume index (OR, 0.95; 95\% CI, 0.91-0.99; $P=$ $0.01)$, LA ejection fraction (OR, 1.06; 95\% CI, 1.02$1.1 ; P=0.005)$, e' mean (OR, $1.29 ; 95 \% \mathrm{CI}, 1.03-$ 1.62; $P=0.03$ ), a' mean (OR, $1.35 ; 95 \% \mathrm{CI}, 1.09$ $1.66 ; P=0.005)$, E wave (OR, 0.07; 95\% CI, 0.01$0.71 ; P=0.02)$, E DT (OR, 1.02; 95\% CI, 1.01-1.03; $P=0.002)$, E/e' mean ratio (OR, $0.83 ; 95 \% \mathrm{CI}, 0.72$ $0.95 ; P=0.007)$, and $\mathrm{E} / \mathrm{A}$ ratio $(\mathrm{OR} 0.54 ; 95 \% \mathrm{CI}$, $0.35-0.84 ; P=0.006)$. The following echocardiographic parameters assessing strain and strain rate were significant predictors of SR: the LASr4c (OR, 1.14; 95\% CI, 1.04-1.25; $P=0.005$ ), LASct4c (OR, 1.45; 95\% CI, 1.179-1.79; $P<0.001$ ), pLASRct4c (OR, 16.53; 95\% CI, 2.95-92.67; $P=0.001$ ), LASr2c (OR, 1.14; 95\% CI, 1.04-1.25; $P=0.003)$, LAScd2c (OR, 1.14; 95\% CI, 1.01-1.29; $P=0.03)$, LASct2c (OR, 1.19; 95\% CI, 1.04-1.36; $P=0.01$ ), and pLASRct2c (OR, 5.65; 95\% CI, 1.77-18.05; $P=0.004)$. The mean values from the $4 c$ and $2 c$ views were also among the significant predictors of SR: LASr mean (OR, 1.18; 95\% CI, 1.06-1.31; $P=0.002)$, LASct mean (OR, 1.34; 95\% CI, 1.12$1.6 ; P=0.002)$, and pLASRct mean (OR, $10.7 ; 95 \%$ CI, 2.44-46.89; $P=0.002$ ).

Multivariable logistic regression analysis that included LA ejection fraction, E/e' mean ratio, LASr $4 c$, and LASct $4 c$ showed that LASct $4 c$ was an independent predictor of SR maintenance during 12-month following DCCV (OR, 1.44, 95\% CI, 1.17-1.77; $P=0.001$ ).

The comparative analysis of the AUCs of the LA strain and strain rate measured in the $4 \mathrm{c}$ view and the average measurements from the $4 c$ and $2 c$ views showed that these parameters had better prognostic properties regarding SR maintenance when measured in the contractile phase than in the reservoir phase (TABLE1).

In the receiver operating characteristic analysis, the following AUCs were obtained for predicting SR maintenance 12 months after DCCV: 0.765 for LASct4c (95\% CI, 0.667-0.863; $P<0.001), 0.68$ for LASr4c (95\% CI, 0.561-0.784; $P=0.003$ ), and 0.726 for pLASRct4c (95\% CI, 0.621-0.831; $P<0.001$ ) (optimal cutoff values, $3.44 \%, 14.55 \%$, $0.39 \mathrm{~s}^{-1}$; sensitivity: $69 \%, 52.4 \%, 73.8 \%$; specificity, $74.5 \%, 78.7 \%, 63.8 \%$; positive predictive value, $70.73 \%, 68.75 \%, 64.58 \%$; and negative predicting value, $72.92 \%, 64.91 \%, 73.17 \%$ for LASct $4 c$, LASr4c, and pLASRct4c, respectively).

Our results show that the LA wall strain and strain rate measured the day after DCCV were significant predictors of SR maintenance after DCCV. Additionally, these parameters had a greater prognostic value when they were measured in the contractile phase than in the reservoir phase. Regarding the strain and strain rate, a $4 \mathrm{c}$ view resulted in the best AUC values for assessing patient prognosis.

Most of the studies published so far on the prognostic value of the LA strain and strain rate have focused on the reservoir phase, which reflects the susceptibility to stretching of the LA muscle but not its contractility. Di Salvo et $\mathrm{al}^{2}$ showed that the strain and strain rate measured before DCCV have a prognostic value in predicting SR maintenance after DCCV in patients with recent-onset, lone $\mathrm{AF}^{2}{ }^{2}$ Wang et $\mathrm{al}^{3}$ demonstrated that the LA strain rate measurement has a prognostic value for SR maintenance after DCCV, but they focused mainly on the basal segments of the left atrium. However, both of these studies were based on the tissue Doppler imaging method. Shaikh et al, ${ }^{4}$ who used the STE technique, found that the predictor of SR maintenance was not the measurement of the LA strain before and after DCCV, but the difference between these values. Morenzo-Ruiz et $\mathrm{al}^{5}$ showed that the LA reservoir strain measured before 
DCCV using the STE technique can be useful in assessing the prognosis of SR maintenance after DCCV. Furthermore, Doruchowa et $\mathrm{al}^{6}$ evaluated the LA strain and the dispersion of time to the LA wall maximum strain, and only the latter had prognostic value in terms of SR maintenance after DCCV. In the studies by Shaikh et $\mathrm{al}^{4}$ and Doruchowska et al, ${ }^{6}$ although strain measurements were taken during SR, no measurements were performed in the LA contractility phase.

Conclusions The LASct4c measurement is a predictor of SR maintenance for 12 months after DCCV. The LASct4c and pLASRct4c have a better prognostic value than these same parameters assessed in the reservoir or conduit phases. Echocardiographic parameters assessing mechanical remodeling better estimate the prognosis of SR maintenance after DCCV than those evaluating structural remodeling.

\section{SUPPLEMENTARY MATERIAL}

Supplementary material is available at www.mp.pl/kardiologiapolska.

\section{ARTICLE INFORMATION}

ACKNOWLEDGMENTS This study was supported by the Ministry of Science and Higher Education, the "Regional Initiative of Excellence" program (no. 024/RID/2018/19; amount granted: 11999000 PLN).

CONFLICT OF INTEREST None declared.

OPEN ACCESS This is an Open Access article distributed under the terms of the Creative Commons Attribution-NonCommercial-NoDerivatives $4.0 \mathrm{In}$ ternational License (CC BY-NC-ND 4.0), allowing third parties to download articles and share them with others, provided the original work is properly cited, not changed in any way, distributed under the same license, and used for noncommercial purposes only. For commercial use, please contact the journal office at kardiologiapolska@ptkardio.pl.

HOW TO CITE Wałek P, Grabowska U, Cieśla E, et al. Left atrial longitudinal strain in the contractile phase as a predictor of sinus rhythm maintenance after electrical cardioversion performed due to persistent atrial fibrillation. Kardiol Pol. 2021; 79: 458-460. doi:10.33963/KP.15913

\section{REFERENCES}

1 Magnani JW, Rienstra M, Lin $\mathrm{H}$, et al. Atrial fibrillation: current knowledge and future directions in epidemiology and genomics. Circulation. 2011; 124: 1982-1989.

2 Di Salvo G, Caso P, Lo Piccolo R, et al. Atrial myocardial deformation properties predict maintenance of sinus rhythm after external cardioversion of recent-onset lone atrial fibrillation: a color Doppler myocardial imaging and transthoracic and transesophageal echocardiographic study. Circulation. 2005; 112: 387-395.

3 Wang T, Wang M, Fung JW, et al. Atrial strain rate echocardiography can predict success or failure of cardioversion for atrial fibrillation: a combined transthoracic tissue Doppler and transoesophageal imaging study. Int J Cardiol. 2007; 114: 202-209.

4 Shaikh AY, Maan A, Khan UA, et al. Speckle echocardiographic left atrial strain and stiffness index as predictors of maintenance of sinus rhythm after cardioversion for atrial fibrillation: a prospective study. Cardiovasc Ultrasound. 2012; 10: 48.

5 Moreno-Ruiz LA, Madrid-Miller A, Martínez-Flores JE, et al. Left atrial longitudinal strain by speckle tracking as independent predictor of recurrence after electrical cardioversion in persistent and long standing persistent non-valvular atrial fibrillation. Int J Cardiovasc Imaging. 2019; 35: 1587-1596.

6 Doruchowska A, Wita K, Bochenek T, et al. Role of left atrial speckle tracking echocardiography in predicting persistent atrial fibrillation electrical cardioversion success and sinus rhythm maintenance at 6 months. Adv Med Sci. 2014; 59: 120-125.

7 Wałek P, Cieśla E, Gorczyca I, Wożakowska-Kapłon B. Left atrial wall dyskinesia assessed during contractile phase as a predictor of atrial fibrillation recurrence after electrical cardioversion performed due to persistent atrial fibrillation. Medicine (Baltimore). 2020; 99: e23333.

8 Badano LP, Kolias TJ, Muraru D, et al. Standardization of left atrial, right ventricular, and right atrial deformation imaging using two-dimensional speckle tracking echocardiography: a consensus document of the EACVI/ASE/Industry Task Force to standardize deformation imaging. Eur Heart J Cardiovasc Imaging. 2018; 19: 591-600. 